\title{
GPS Output Signal Processing considering both Correlated/White Mea- surement Noise for Optimal Navigation Filtering
}

\author{
Do-Myung Kim* and Jinyoung Suk** \\ Chungnam National University, 99 Daehak-ro, Yuseong-gu, Daejeon 305-764, Republic of Korea
}

\begin{abstract}
In this paper, a dynamic modeling for the velocity and position information of a single frequency stand-alone GPS(Global Positioning System) receiver is described. In static condition, the position error dynamic model is identified as a first/second order transfer function, and the velocity error model is identified as a band-limited Gaussian white noise via non-parametric method of a PSD(Power Spectrum Density) estimation in continuous time domain. A Kalman filter is proposed considering both correlated/white measurements noise based on identified GPS error model. The performance of the proposed Kalman filtering method is verified via numerical simulation.
\end{abstract}

Key words: GPS Error Modeling, Power Spectral Density, Correlated Measurement Noise, Kalman filter.

\section{Introduction}

GPS has been used as main navigation system for a variety of aerospace applications. Kalman filter estimation is widely applied to GPS positioning as well as the integration of GPS with inertial navigation system. A conventional Kalman filter is formulated with assumption that both the process and measurement noise are Gaussian white. There are some GPS measurement error modeling studies under SA(Selective Availability) condition[1-4]. Where GPS position measurement error can be modeled as a time correlated low order Gauss-Markov process both continuous and discrete time domain using various parameter identification approaches. Because it is difficult to determine the parameters of Gauss-Markov process in a real-field problem, it is important to estimate of the amount of experimental data recoding time and ensemble average needed for a given required estimation accuracy.

Previous works for kalman filtering methods that consider time-correlated measurement error are categorized in two main approaches. First approach is state augmentation method where the measurement error is augmented into state vector. However, the augmented sate space equation has the perfect measurement with singular measurement covariance matrix that may cause system to become ill-conditioned[5-7]. The second approach uses measurement differencing method to make a new measurement equation corrupted by white noise without time-correlated part[6-9]. Therefore the conventional Kalman filter can be applied to this state and new measurement equation for optimal state estimation. However, In case of multiple measurements, the measurement differencing approach is limited by each measurement should be simultaneously time-correlated.

This paper describes GPS error modeling method for stand-alone single frequency GPS. The relationship between required data recoding time and ensemble average number to satisfy the desired PSD(Power Spectrum Density) estimation error is investigated. It is also shown that the physical parameter of a first or second order dynamic system is related with required data recoding time and ensemble average number. A Kalman filtering method is proposed that consider both correlated/white measurements noise based on characteristics of a stand-alone GPS position and velocity measurements. The performance of the proposed Kalman filtering method is verified via numerical simulation.
This is an Open Access article distributed under the terms of the Creative Commons Attribution Non-Commercial License (http://creativecommons.org/licenses/by$\mathrm{nc} / 3.0 /$ ) which permits unrestricted non-commercial use, distribution, and reproduction in any medium, provided the original work is properly cited. * Graduate Student, Department of Aerospace Engineering
** Associate Professor, Department of Aerospace Engineering
Corresponding author: jsuk@cnu.ac.kr 


\section{GPS Error Modeling}

\subsection{Linear System and PSD}

The relationship between PSD and transfer function of single input-output linear time invariant system is described as follows[10].

$$
S_{x x}(\omega)=|G(j \omega)|^{2} S_{u u}(\omega)
$$

where $G(w)$ is a transfer function of the system, $S_{u u}(w)$ is the PSD of input, and $S_{x x}(w)$ is the PSD of output. The time average of the periodogram of a random process $x(t)$ can be expressed as follows[7].

$$
\lim _{T \rightarrow \infty} \frac{1}{T}\left|\mathfrak{I}\left\{x_{T}(t)\right\}\right|^{2}=\int_{-\infty}^{\infty} R_{x x}(\tau) e^{-j \omega \tau} d \tau=S_{x x}(\omega)
$$

where $x_{T}(t)$ is a time sample of the random process $x(t)$ at time $t, \mathfrak{I}\left[x_{T}(t)\right]$ is Fourier transform of the $x_{T}(t)$. If $S_{u u}(w)$ is unit power white noise and $S_{x x}(w)$ is the error signal of a GPS, using equation (1) and (2), we can find transfer function of the GPS error model with experimental data of $S_{x x}(w)$.

\subsection{Normalized PSD Estimation Error}

The normalized bias error of PSD estimation can be approximated via Taylor series expansion of expectation of the PDF(Probability Density Function) of a random process $x(t)[11]$.

$$
\varepsilon_{b}\left[\widehat{S_{x x}}(\omega)\right] \approx\left(\frac{W^{2}}{24}\right)\left(\frac{\ddot{S}(\omega)}{S(\omega)}\right)
$$

where $W$ is the resolution bandwidth. Eq. (3) can be rearranged as

$$
W \approx \sqrt{24 \varepsilon_{b}\left[\widehat{S_{x x}}(\omega)\right]\left(\frac{S_{X}(\omega)}{\ddot{S}_{X}(\omega)}\right)}
$$

The normalized random error of a PSD estimation can be represented using identity that the two degree of freedom chi-square variables have average $n$ and variance $2 n$.

$$
\mathcal{E}_{r}\left[\widehat{S_{x x}}(\omega)\right]=\frac{1}{\sqrt{n_{d}}}
$$

where $n_{d}$ is ensemble average number. The required data recoding time for given normalized random error is

$$
T_{r}=\frac{2 \pi}{W \varepsilon_{r}^{2}\left[\widehat{S_{x x}}(\omega)\right]}
$$

\subsection{Transfer Function Parameter and PSD}

An arbitrary transfer function and PSD of the first-order/ second-order Gauss Markov process can be represented as follows.

First order:

$$
\begin{aligned}
& G(s)=\frac{k}{1+T s} \\
& S(\omega)=\frac{(k / T)^{2}}{\omega^{2}+(1 / T)^{2}}
\end{aligned}
$$

Second order:

$$
\begin{aligned}
& G(s)=\frac{a s+b}{s^{2}+2 \zeta \omega_{n} s+\omega_{n}^{2}} \\
& S(\omega)=\frac{a^{2} \omega^{2}+b^{2}}{\omega^{4}+2 \omega_{n}^{2}\left(2 \zeta^{2}-1\right) \omega^{2}+\omega_{n}^{4}}
\end{aligned}
$$

To investigate the relationship between PSD estimation error and resolution bandwidth, we define $S R \cong \frac{\ddot{S}(\omega)}{S(\omega)}$ and conservative approach should be applied to determine the frequency for calculation of in Eq. (4).

As shown in the Fig. 1. (a) (b), $S F$ of the first-order Gauss-Markov reaches maximum value at the frequency $\omega_{s}=\sqrt{15} / 3 T$, and the second-order Gauss-Markov process has an extreme value of $S F$ depending on the damping ratio. In case of a second-order Gauss-Markov process with lightly damped system(damping ratio range is $\mathrm{C}<0.3$ ), has a maximum value at the resonance frequency $\omega_{n} \sqrt{1-2 \zeta \omega_{n}}$. In case of normally damped system, that has a damping ratio is $0.3<\mathrm{C}<0.707$, has a maximum value at the damped natural frequency $\omega_{d}=\omega_{n} \sqrt{1-\zeta \omega_{n}}$. In case of over damped system, that has a damping ratio is $\mathrm{C}>0.707, S F$ has a maximum value at the frequency $\omega_{m}=2 \pi / T$. . Therefore, $W$ should be evaluated at the appropriate frequency for given PSD estimation error bound based on damping ratio of the second-order linear system.

\subsection{Estimation of the PSD and Transfer Function}

In static condition, the position error dynamic model is identified as a first/second order transfer function, and the velocity error model is identified as a band-limited Gaussian white noise via non-parametric method of a PSD estimation in continuous time domain. Based on quick identification of the position error model of the second order transfer function and its damping ratio $w_{m}$ using one hour recoding data analysis, we obtained $n_{d}=25$ and $T_{r}=45$ minutes that satisfy PSD estimation error boundary $\varepsilon_{b}\left[\widehat{S_{x x}}(\omega)\right]=0.01$ and $\varepsilon_{r}\left[\widehat{S_{x x}}(\omega)\right]=0.2$.

Fig. 2. (a) shows effectiveness of ensemble average of the PSD in frequency domain. In this case, 25 data set of 


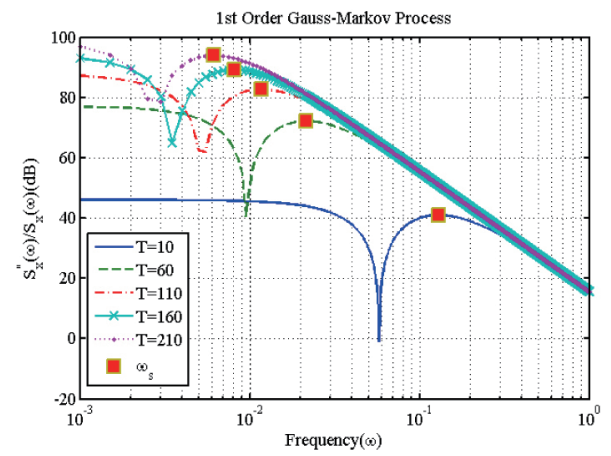

(a)

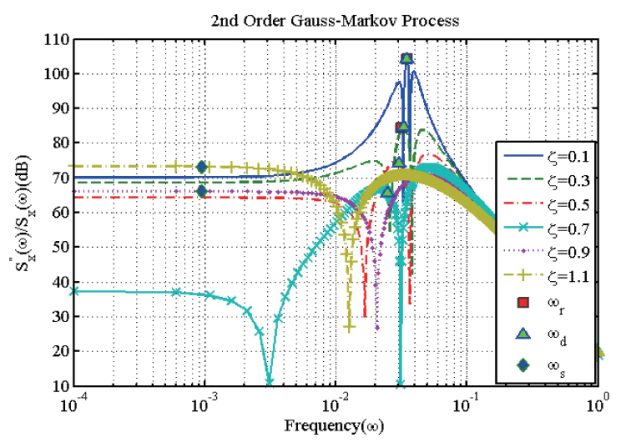

(c)

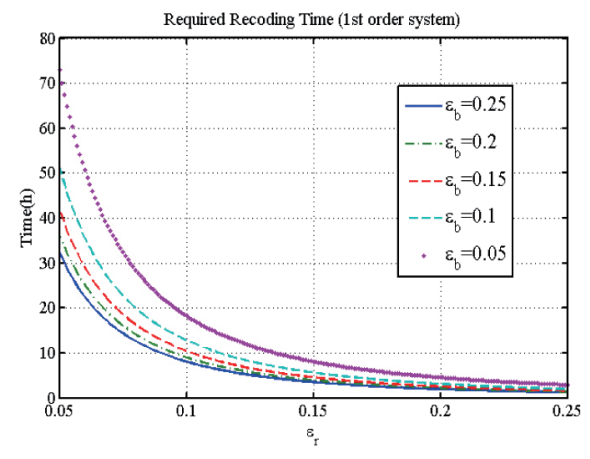

(b)

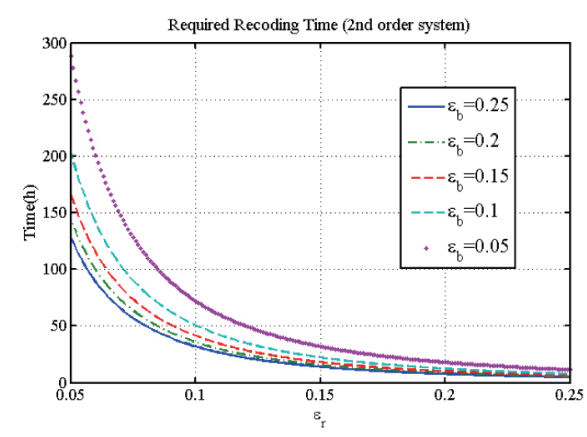

(d)

Fig. 1. Transfer Function Parameter and Required Recoding Time: (a) First Order System SR with respect to Time Constant, (b) First Order System Required Recoding Time with respect to Bias Error, (c) Second Order System SR with respect to Damping Ratio, (d) Second Order System Required Recoding Time with respect to Bias Error.

position error PSD are averaged. Fig. 2. (b) shows fitting of the averaged PSD estimation using nonlinear least square regression method. Fig. 2. (c) bode diagram of the identified transfer function. Fig. 2. (d) shows comparison of the real experimental data and linear simulation of the identified transfer function. In fact, the GPS position error model can be approximated as a first order transfer function within low frequencies with $-20 \mathrm{~dB} / \mathrm{dec}$ roll-off ratio of the magnitude as shown in Fig. 2. (c).

The Identified GPS position error model in NEU coordinate system is summarized in the Table 1.

As shown in the Fig. 3, the velocity measurement error PSD has relatively low roll-off ratio and flat magnitude within Nyquist frequency. Therefore it is reasonable to assume that velocity measurement noise is band limited white.

A Kalman filter can be applied to the state-space equation transformed from the error transfer function. The first order transfer function can be obtained as Eq.(11). $\sigma$ and $\delta$ of the first order transfer function corresponding to each axis on NEU coordinate system is summarized in Table 2.

$$
\dot{x}(t)=-\beta \cdot x(t)+\sqrt{2 \sigma^{2} \beta} \cdot u(t)
$$

Eq.(11) can be discretized for the sampling time $\Delta t$ yielding

$$
x(k+1)=e^{-\beta \Delta t} x(k)+\frac{1}{\beta} \sqrt{2 \sigma^{2} \beta}\left(1-e^{-\beta \Delta t}\right) \cdot u(k)
$$

\section{Kalman Filtering Methodology}

The Kalman filtering method is applied for one measurement noise is first order Gauss-Markov process and the other measurement noise is white. Consider the discrete state and measurement equation with both colored measurement noise and white noise.

$$
\begin{aligned}
& x(k+1)=\Phi x(k)+w(k) \\
& z(k)=C x(k)+e(k)+d(k) \\
& e_{r}(k+1)=\psi e_{r}(k)+v_{r}(k) \\
& w(k) \sim(0, Q) \\
& d(k) \sim(0, D)
\end{aligned}
$$




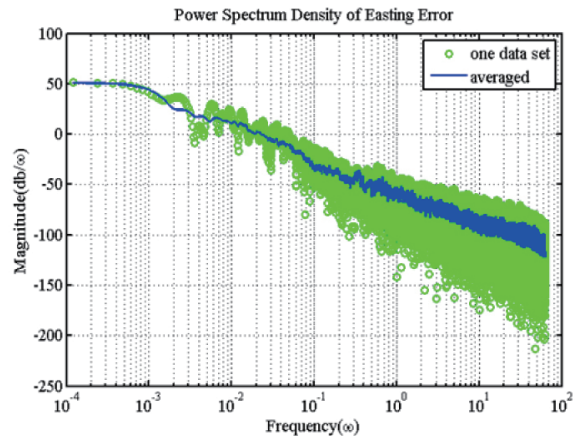

(a)

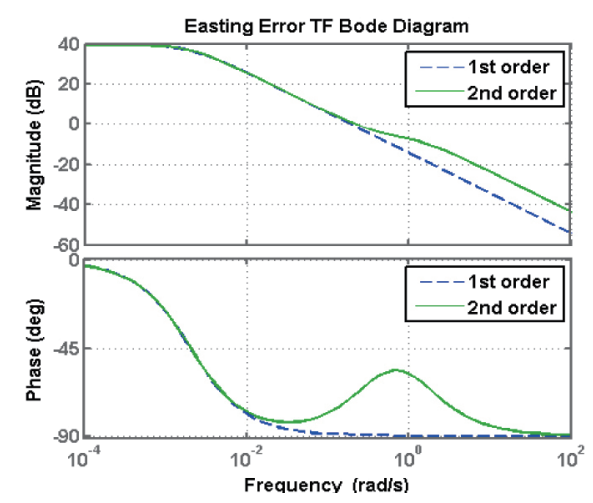

(c)

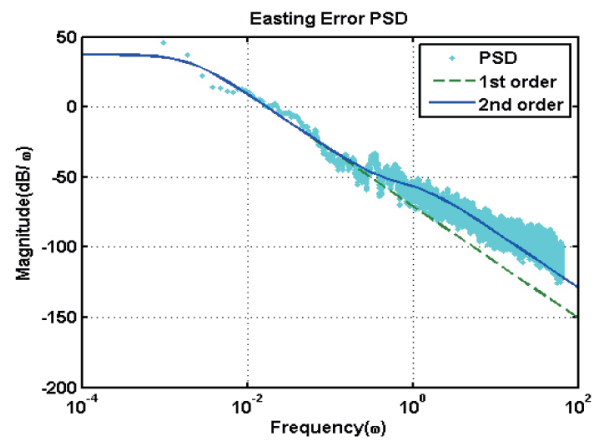

(b)
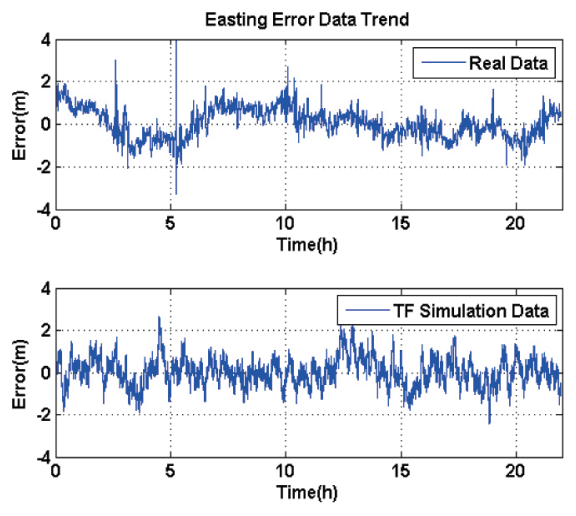

(d)

Fig. 2. Identification of the Easting Position Error Transfer Function: (a) Ensemble Average, (b) Nonlinear Least Square Fitting with respect to PSD, (c) Bode Diagram of the Identified Transfer Function, (d) Comparison Real Data and Linear Simulation of ID

Table 1. Summary of the GPS Position Error Model

\begin{tabular}{|c|c|c|c|c|}
\hline & & Northing & Easting & Up \\
\hline \multirow[t]{2}{*}{$\begin{array}{l}1^{\text {st }} \\
\text { Order }\end{array}$} & $S(\omega)=\frac{2 \sigma^{2} \beta}{\omega^{2}+\beta^{2}}$ & $\frac{0.07531}{\omega^{2}+1.456 e-006}$ & $\frac{0.03659}{\omega^{2}+4.006 e-006}$ & $\frac{0.3829}{\omega^{2}+2.571 e-006}$ \\
\hline & $G(s)=\frac{\sqrt{2 \sigma^{2} \beta}}{s+\beta}$ & $\frac{0.2744}{s+0.001207}$ & $\frac{0.1913}{s+0.002002}$ & $\frac{0.6188}{s+0.001604}$ \\
\hline \multirow[t]{2}{*}{$\begin{array}{l}2^{\text {nd }} \\
\text { Order }\end{array}$} & $S(\omega)=\frac{a^{2} \omega^{2}+b^{2}}{\omega^{4}+2 \omega_{t}^{2}\left(s^{2}-1\right) \omega^{2}+\omega_{x}^{4}}$ & $\frac{132 \omega^{2}+1.674}{\omega^{4}+22222 \omega^{2}+0.0001169}$ & $\frac{0.441 \omega^{2}+0.06153}{\omega^{4}+1.682 \omega^{2}+6.737-0.066}$ & $\frac{7.327 \sigma^{2}+9.748}{a^{4}+25.46 \omega^{2}+6.5477-0.05}$ \\
\hline & $G(s)=\frac{a s+b}{s^{2}+2 \varsigma \omega_{n} s+\omega_{n}{ }^{2}}$ & $\frac{1.149 s+1.294}{s^{2}+4.716 s+0.01081}$ & $\frac{0.6641 s+0.2481}{z^{2}+1.2998+0.002596}$ & $\frac{2.707 s+3.122}{s^{2}+5.048 s+0.008091}$ \\
\hline
\end{tabular}

Table 2. Summary of the Model Parameters of 1st Order Transfer Function

\begin{tabular}{|l|c|c|}
\hline & $\sigma$ & $\beta$ \\
\hline Northing & 4.053 & 0.0023 \\
\hline Easting & 3.023 & 0.0020 \\
\hline Up & 10.93 & 0.0016 \\
\hline
\end{tabular}

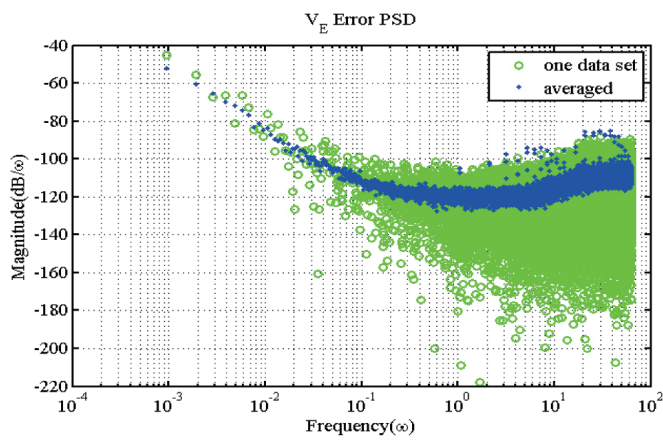

Fig. 3. Ensemble Average of 25 Data Set of Velocity Error PSD.

$$
v_{c}(k) \sim(0, V)
$$

where $x \in R^{n_{x}}, z \in R^{n_{z}}, e \in R^{n_{e}}, w, d, c$ denotesstates, measurements, state of time-time correlated measurement error, process noise, white noise measurement and white noise for shaping time-correlated measurement error, respectively. These white noises are mutually un-correlated. State variables can be classified as the three groups:(i) $x_{c}$, which are disturbed 
Do-Myung Kim GPS Output Signal Processing considering both Correlated/White Measurement Noise for Optimal Navigation...

by the time-correlated measurement noise, (ii) $x_{n}$, which are disturbed by white measurement noise, and (iii) $x_{s}$, which can be estimated. Each state variables have rows $n_{c}, n_{n}$ and $n_{s}$. It is also assumed that the time-correlated measurement noise can be represented by the first order transfer function having $n_{c}$ state variables. Eq.(19) shows the size of each variable vectors with appropriate subsystems.

$$
C=\underbrace{\left[\begin{array}{lll}
I & 0 & 0 \\
0 & I & 0
\end{array}\right]}_{n_{c}} \underbrace{[}_{n_{s}}\} n_{c}
$$

State and measurement equations can be rewritten using Eq.(19) as

$$
\begin{gathered}
{\left[\begin{array}{c}
x_{c}(k+1) \\
x_{n}(k+1) \\
x_{s}(k+1)
\end{array}\right]=\left[\begin{array}{c}
\Phi_{11} \Phi_{12} \Phi_{13} \\
\Phi_{21} \Phi_{22} \Phi_{23} \\
\Phi_{31} \Phi_{32} \Phi_{33}
\end{array}\right]\left[\begin{array}{c}
x_{c}(k) \\
x_{n}(k) \\
x_{s}(k)
\end{array}\right]+\left[\begin{array}{c}
w_{c}(k) \\
w_{n}(k) \\
w_{s}(k)
\end{array}\right]} \\
{\left[\begin{array}{c}
z_{c}(k) \\
z_{n}(k)
\end{array}\right]=\left[\begin{array}{lll}
I & 0 & 0 \\
0 & I & 0
\end{array}\right]\left[\begin{array}{c}
x_{c}(k) \\
x_{n}(k) \\
x_{s}(k)
\end{array}\right]+\left[\begin{array}{c}
e_{c}(k) \\
0
\end{array}\right]+\left[\begin{array}{c}
0 \\
d_{n}(k)
\end{array}\right]}
\end{gathered}
$$

After augmenting time-correlated measurement error state into state vector, state and measurement equations can be rearranged as follows.

$$
\begin{aligned}
& x_{a}(k+1)=\Phi_{a} x_{a}(k)+w_{a}(k) \\
& z(k)=C_{a} x(k)+d_{a}(k)
\end{aligned}
$$

where,

$$
\begin{aligned}
& x_{a}(k)=\left[\begin{array}{l}
x_{c}(k) \\
x_{n}(k) \\
x_{s}(k) \\
e_{c}(k)
\end{array}\right], w_{a}(k)=\left[\begin{array}{c}
w_{c}(k) \\
w_{n}(k) \\
w_{s}(k) \\
v_{c}(k)
\end{array}\right], \\
& \Phi_{a}=\left[\begin{array}{ll}
\Phi & 0 \\
0 & \Psi
\end{array}\right], \quad C_{a}=\left[\begin{array}{llll}
I & 0 & 0 & I \\
0 & I & 0 & 0
\end{array}\right], d_{a}=\left[\begin{array}{c}
0 \\
d_{n}(k)
\end{array}\right]
\end{aligned}
$$

As can be seen in Eq.(24), time-correlated measurement for augmented system model does not contain the white noise. A numerical difference method is used in this paper to avoid the 'perfect measurement condition', which is often the cause of numerical instability. In order to do this, a new measurement vector can be defined as the linear difference of the measurement $z$ between $t(k-1)$ and $t(k)$ as follows.

$$
y=\left[\begin{array}{c}
y_{c} \\
y_{n}
\end{array}\right] \cong\left[\begin{array}{c}
z_{c}(k)-\Psi z_{c}(k-1) \\
z_{n}(k)
\end{array}\right]
$$

Combination of the augmented state equation and the new measurement equation yields

$$
y_{c}(k)=z_{c}(k)-\Psi z_{c}(k-1)
$$

$z_{c}(k)=x_{c}(k)+e_{c}(k)$

$z_{c}(k)=\left[\Phi_{11}-\Psi\right] x_{c}(k-1)+\Phi_{12} x_{n}(k-1)+\Phi_{13} x_{s}(k-1)+w_{c}(k-1)(28)$

$e_{c}(k)=\Psi e_{c}(k-1)+v_{c}(k-1)$

Using Eqs(28)-(29) Eq.(30) is obtained.

$z_{c}(k)=\Phi_{11} x_{c}(k-1)+\Phi_{12} x_{n}(k-1)+\Phi_{13} x_{s}(k-1)+w_{c}(k-1)+\Psi_{e}(k-1)+v_{c}(k-1)$

Eq.(27) can be rearranged as,

$e_{c}(k-1)=z_{c}(k-1)-x_{c}(k-1)$

Eqs.(30)-(31) yields,

$$
z_{c}(k)=\left[\Phi_{11}-\Psi\right] x_{c}(k-1)+\Phi_{12} x_{n}(k-1)+\Phi_{13} x_{s}(k-1)+w_{c}(k-1)+\Psi z_{c}(k-1)+v_{c}(k-1)
$$

Using Eq.(26) and Eq(32), expression on $u_{c}(k)$ can be given by

$$
y_{c}(k)=\left[\begin{array}{llll}
\Phi_{11}-\Psi & \Phi_{12} & \Phi_{13} & 0
\end{array}\right] x_{a}(k-1)+w_{c}(k-1)+v_{c}(k-1)
$$

Time delay of the update on state estimation can be avoided when the measurement can be rewritten using the inverse of the state transition matrix of the augmented state equations:

$$
\begin{aligned}
& \Phi_{a}^{-1}\left[x_{a}(k)-w_{a}(k-1)\right]=x_{a}(k-1)
\end{aligned}
$$

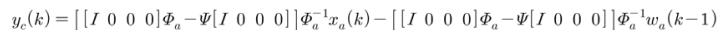

$$
\begin{aligned}
& y_{n}(k)=\left[\begin{array}{llll}
0 & I & 0 & 0
\end{array}\right] \Phi_{a}-\Psi\left[\begin{array}{llll}
0 & 0 & 0 & 0
\end{array}\right]\left[\Phi_{a}^{-1} x_{a}(k)+d(k)\right.
\end{aligned}
$$

Now, new parameters $N$ and $U$ are introduced to combine Eqs.(35)-(36),

$y(k)=\left[C_{a}-\Psi \cdot N \cdot C_{a} \cdot \Phi_{a}^{-1}\right] x_{a}(k)+\Psi \cdot N \cdot C_{a} \cdot \Phi_{a}^{-1} \cdot w_{a}(k-1)+\left[\begin{array}{c}v_{c}(k-1) \\ 0\end{array}\right]+\left[\begin{array}{c}0 \\ d(k)\end{array}\right](37)$

where $N=\underbrace{\left[\begin{array}{ll}I & 0 \\ 0 & 0\end{array}\right]}_{n_{c}}\} n_{n_{n}}\} n_{c}, U=\underbrace{\left[\begin{array}{l}0 \\ 0\end{array}\right]}_{i}\} n_{n} n_{c}, C_{a}=\left[\begin{array}{ll}C & U\end{array}\right]$

Eq.(22) and Eq.(37) can be simplified as,

$$
\begin{aligned}
& x_{a}(k)=\Phi_{a} x_{a}(k-1)+w_{a}(k-1) \\
& y(k)=H x_{a}(k)+s(k) \\
& H=\left[C_{a}-\Psi \cdot N \cdot C_{a} \cdot \Phi_{a}^{-1}\right] \\
& s(k)=\Psi \cdot N \cdot C_{a} \cdot \Phi_{a}^{-1} \cdot w_{a}(k-1)+\left[\begin{array}{c}
v_{c}(k-1) \\
0
\end{array}\right]+\left[\begin{array}{c}
0 \\
d(k)
\end{array}\right]
\end{aligned}
$$

where the new measurement noise is white, and $w_{a}(k-1)$, $w_{c}(k-1), d(k)$ are not time-correlated. However, the process noise and measurement noise is correlated because both state equation and measurement equation have the term $w_{a}(k-1)$. Measurement noise covariance matrix $F$ and 
process-measurement cross covariance matrix $S$ can be defined as,

$$
\begin{aligned}
R= & E\left\{s(k) s(k)^{T}\right\} \\
= & E\left[\left(\Psi \cdot N \cdot C_{a} \cdot \Phi_{a}^{-1} \cdot w_{a}(k-1)+\left[\begin{array}{c}
v_{c}(k-1) \\
0
\end{array}\right]+\left[\begin{array}{c}
0 \\
d(k)
\end{array}\right]\right) \cdot\left(w_{a}(k-1)^{T} \cdot \Phi_{a}^{-T} \cdot C_{a}^{T} \cdot N^{T} \cdot \Psi^{T}\right)\right\} \\
& +E\left\{\left[\begin{array}{c}
v_{c}(k-1) \\
0
\end{array}\right]^{T}+\left[\begin{array}{c}
0 \\
d(k)
\end{array}\right]^{T}\right\} \\
= & \Psi \cdot N \cdot C_{a} \cdot \Phi_{a}^{-1} \cdot Q_{a}(k-1) \cdot \Phi_{a}^{-T} \cdot C_{a}^{T} \cdot N^{T} \cdot \Psi^{T}+\left[\begin{array}{cc}
V & 0 \\
0 & 0
\end{array}\right]+\left[\begin{array}{cc}
0 & 0 \\
0 & D
\end{array}\right] \\
S & =E\left\{w_{a}(k-1) \cdot s(k)^{T}\right\} \\
= & E\left\{w_{a}(k-1) \cdot w_{a}(k-1)^{T}\right\} \Phi_{a}^{-T} \cdot C_{a}^{T} \cdot N^{T} \cdot \Psi^{T}+E\left\{w_{a}(k-1) \cdot\left[\begin{array}{c}
v_{c}(k-1) \\
0
\end{array}\right]^{T}\right\} \\
= & Q_{a} \cdot \Phi_{a}^{-T} \cdot C_{a}^{T} \cdot N^{T} \cdot \Psi^{T}+\left[\begin{array}{cc}
0 & 0 \\
0 & 0 \\
0 & 0 \\
V & 0
\end{array}\right]
\end{aligned}
$$

In general, Kalman filter cannot be applied for the case process noise and measurement noise is correlated. Therefore, a generalized Kalman filter is used in this paper in order to obtain optimal estimation that considers both time-correlated measurement noise and white noise. State equation and measurement equation with updates for a generalized Kalman filter are shown in Eq.(44)-(53)[12].

$$
\begin{aligned}
& x_{a}(k)=\Phi_{a} x_{a}(k-1)+w_{a}(k-1) \\
& y(k)=H x_{a}(k)+s(k) \\
& E\left[w_{a}(j) w_{a}^{T}(k)\right]=Q_{a} \delta_{j k}
\end{aligned}
$$

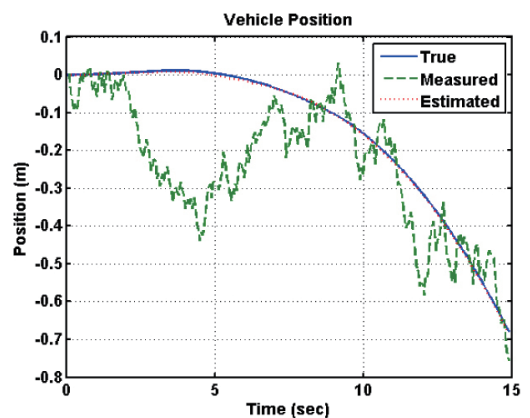

(a)

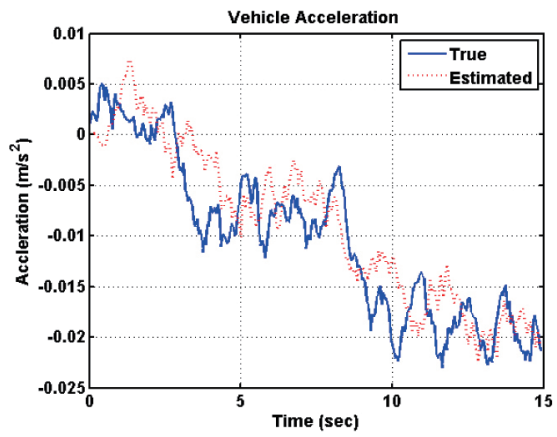

(c)
$E\left[s(j) s^{T}(k)\right]=R \delta_{i k}$

$$
E\left[w_{a}(j-1) s^{T}(k)\right]=S \delta_{(j-1) k}
$$

Time Update

$$
\begin{aligned}
& \hat{x}_{a}^{-}(k)=\Phi_{a} \hat{x}_{a}^{+}(k-1) \\
& P^{-}(k)=\Phi_{a} P^{+}(k-1) \Phi^{T}+Q_{a}
\end{aligned}
$$

Measurement Update

$$
\begin{aligned}
& K(k)=\left(P^{-}(k) H^{T}+S\right)\left(H P^{-}(k) H^{T}+H S+S^{T}+R\right)^{-1} \\
& \hat{x}_{a}^{+}(k)=\hat{x}_{a}^{-}(k)+K(k)\left(y(k)-H \hat{x}_{a}^{-}(k)\right) \\
& P^{+}(k)=P^{-}(k)-K(k)\left(H P^{-}(k)+S^{T}\right)
\end{aligned}
$$

\section{Numerical Simulation}

A simple kinematic CWNJM(Continuous White Noise Jerk Model) is considered for numerical simulation. The states are position, velocity and acceleration. It is assumed that the position measurement noise is first-order GaussMarkov process and velocity measurement noise is white as

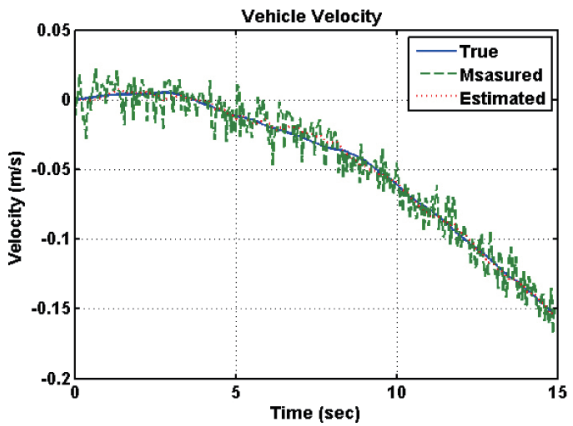

(b)

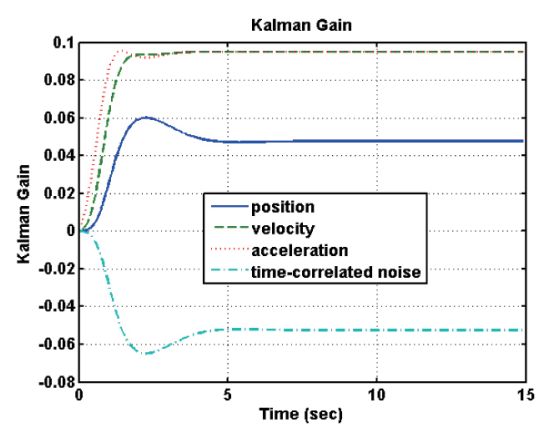

(d)

Fig. 4. Simulation Results: (a) Position Estimation, (b) Velocity Estimation, (c) Acceleration Estimation, (d) Kalman Gain. 
mentioned in chapter II. Discretized linear dynamic model of CWNJM as follows.

$$
\begin{aligned}
& x(k)=\Phi x(k-1)+w(k-1) \\
& z(k)=C x(k)+e_{c}(k)+d(k) \\
& e_{c}(k+1)=\psi e_{c}(k)+v_{c}(k) \\
& w(k) \sim(0, Q) \\
& d(k) \sim(0, D) \\
& v(k) \sim(0, V) \\
& E\left[w(j) w^{T}(k)\right]=Q \delta_{j k} \\
& E\left[d(j) d^{T}(k)\right]=D \delta_{j k} \\
& E\left[v_{c}(j) v_{c}^{T}(k)\right]=V \delta_{j k}
\end{aligned}
$$

where,

$$
\begin{aligned}
& x=\left[\begin{array}{c}
x_{c}(\text { Position }) \\
x_{n}(\text { Velocity }) \\
x_{s}(\text { Acceleration })
\end{array}\right], \Phi=\left[\begin{array}{ccc}
1 & T & \frac{1}{2} T \\
0 & 1 & T \\
0 & 0 & 1
\end{array}\right], \\
& Q=\left[\begin{array}{ccc}
\frac{1}{20} T^{5} & \frac{1}{8} T^{4} & \frac{1}{6} T^{3} \\
\frac{1}{8} T^{4} & \frac{1}{3} T^{3} & \frac{1}{2} T^{2} \\
\frac{1}{6} T^{3} & \frac{1}{2} T^{3} & T
\end{array}\right] q,, \quad z=\left[\begin{array}{l}
z_{c} \\
z_{n}
\end{array}\right], \quad C=\left[\begin{array}{lll}
1 & 0 & 0 \\
0 & 1 & 0
\end{array}\right]
\end{aligned}
$$

State equation for augmented system can be expressed as,

$$
x_{a}(k)=\Phi_{a} x_{a}(k-1)+w_{a}(k-1)
$$

where $x_{a}(k)=\left[\begin{array}{c}x_{c}(k) \\ x_{n}(k) \\ x_{s}(k) \\ e_{c}(k)\end{array}\right], w_{a}(k)=\left[\begin{array}{c}w_{c}(k) \\ w_{n}(k) \\ w_{s}(k) \\ v_{c}(k)\end{array}\right], \Phi_{a}=\left[\begin{array}{cc}\Phi & 0 \\ 0 & \Psi\end{array}\right]$.

Discretized version of measurement equation can be given by the following equations

$$
\begin{aligned}
& y(k)=H x_{a}(k)+s(k) \\
& y(k) \cong\left[\begin{array}{c}
z_{c}(k)-\Psi z_{c}(k-1) \\
z_{n}(k)
\end{array}\right] \\
& H=\left[C_{a}-\Psi \cdot N \cdot C_{a} \cdot \Phi_{a}^{-1}\right] \\
& R=\Psi \cdot N \cdot C_{a} \cdot \Phi_{a}^{-1} \cdot Q_{a}(k-1) \cdot \Phi_{a}^{-T} \cdot C_{a}^{T} \cdot N^{T} \cdot \Psi^{T}+\left[\begin{array}{ll}
V & 0 \\
0 & 0
\end{array}\right]+\left[\begin{array}{ll}
0 & 0 \\
0 & D
\end{array}\right] \\
& S=Q_{a} \cdot \Phi_{a}^{-T} \cdot C_{a}^{T} \cdot N^{T} \cdot \Psi^{T}+\left[\begin{array}{ll}
0 & 0 \\
0 & 0 \\
0 & 0 \\
V & 0
\end{array}\right]
\end{aligned}
$$

Now the state equation and measurement equation are reconstructed so that the generalized Kalman filter can be applied. Parameters for process noise and white noise are set as $q=0.001^{2}, D=0.01^{2}, V=0.03^{2} . \Psi=0.999925$ for Northing position error model from the discretization of the GPS position error model with $\triangle t=0.05$ in Eq.(12). Numerical simulation is performed using Eqs.(44)-(53).

Fig. 4. (a) and (b) shows the proposed filter effectively estimates both position corrupted by time-correlated measurement error and velocity corrupted by white noise. Also, the acceleration estimation is appropriate as shown in Fig. 4 (c). In this example, the process noise covariance is set relatively small value to show the estimate with respect to noise magnitude during small time window. It should be noted that the initial states are assumed to be known. Actually, this proposed Kalman filter de-correlates the time correlated measurement error of the position using velocity information which is not corrupted by time correlated noise, but by white noise. Moreover, using the velocity information and simple kinematics, we can get acceleration estimation without additional measurements.

\section{Conclusions}

In this paper, a dynamic modeling method for the velocity and position information of a single frequency stand-alone GPS receiver is described. The relationship between ensemble averages, required data recoding time and PSD estimation error that satisfy the given error bound is described. Also, analysis on transfer function parameters of a first and a second order linear system with respect to PSD error is described. A Kalman filter is proposed that consider both correlated/white measurements noise based on identified GPS error model. The proposed Kalman filter is derived from the fusion of the state augmentation approach and measurement differencing approach. The performance of the proposed Kalman filter is verified via numerical simulation. Using this filter, the time correlated position error of the GPS measurement is effectively decorrelated via its own GPS velocity information without any additional sensors. In near future, the proposed Kalman filtering method will be formulated for more general cases and applied to the precise navigation of moving vehicles.

\section{Acknowlegments}

This study was financially supported by research fund of Chungnam National University in 2010. 


\section{References}

[1] Change No. 1 to RTCA/DO-208, RTCA paper no. 47993/TMC-106, RTCA Inc.,Washington, DC, 1993.

[2] Y. Bian, "GPS signal selective availability modeling and simulation for FAA WAAS IV\&V," ION GPS-95, pp. 761-769, 1995.

[3] X. Kong, “GPS Modeling in Frequency Domain," Proc. of the 2nd International Conference on Wireless broadband and Ultra Wideband Communications, 2007.

[4] S. Khoomboon, T. Kasetkasem, R. Keinprasit, N. Sugino, "Increase a standalone GPS positioning accuracy by using a proximity sensor," Proc. of the International Conference on ECTI-CON, pp. 584-587, 2010.

[5] A. Gelb "Applied optimal estimation," The MIT Press, Cambridge, 1974.

[6] A. E. Jr. Bryson, L. J. Henrikson "Estimation using sampled data containing sequentially correlated noise," Journal of Spacecraft and Rockets, Vol. 5, Issue 6, pp. 662-
665, 1968.

[7] R. G. Brown and P. Y. C. Hwang, Introduction to Random Signals and Applied Kalman Filtering, Second Edition, John Wiley \& Sons, Inc, 1992

[8] M. G. Petovello, K. O'Keefe, G. Lachapelle, M. E. Cannon, "Consideration of time-correlated errors in a Kalman filter applicable to GNSS," Journal of Geodesy, Volume 83, Issue 1, pp. 51-56, 2009.

[9] R. GAZIT, "Digital Tracking Filters with High Order Correlated Measurement Noise," IEEE Transactions on Aerospace and Electronic Systems, Vol. 33, Issue 1, pp. 171177, 1997.

[10] J. S. Kim, Linear Control System Engineering, Choungmoongak, Inc, 2001.

[11] J. S. Bendat and A. G. Piersol, Random Data: Analysis \& Measurement Procedures, Third Edition, John Wiley \& Sons, Inc, 1986.

[12] D. Simon, Optimal State Estimation, WileyInterscience, 2006. 